\title{
Effects of using a peer support system to optimise knowledge sharing in learning networks: a cognitive load perspective
}

\author{
Ya Ping Hsiao*, Francis Brouns and \\ Peter B. Sloep
}

Research Centre for Learning, Teaching and

Technology (Welten Institute),

Open University of the Netherlands,

P.O. Box 2960, 6401 DL, Heerlen, The Netherlands

Email: amy.hsiao@ou.nl

Email: francis.brouns@ou.nl

Email: peter.sloep@ou.nl

*Corresponding author

\begin{abstract}
In a non-formal learning network, knowledge sharing is often desirable when working on complex tasks. However, without support, learners need to first find a tutor and then maintain social interaction, which, according to cognitive load theory, may hamper learning. After all, the extraneous load imposed by these two activities and the intrinsic load imposed by the task itself might easily overload learners' cognitive capacity. We compared the effects of using a peer support system with an automatic tutor assignment and an interaction tool (wiki) to a forum and control group (without any support) on learners' cognitive load and learning efficiency for simple and complex tasks. The results did not significantly show that this peer support system was instrumental in reducing cognitive load and improving learning efficiency. However, the study did shed an illuminating light on how to apply instructional guidelines of cognitive load theory to non-formal learning networks.
\end{abstract}

Keywords: knowledge sharing; peer tutoring; peer support system; PSS; cognitive load; learning networks.

Reference to this paper should be made as follows: Hsiao, Y.P., Brouns, F. and Sloep, P.B. (xxxx) 'Effects of using a peer support system to optimise knowledge sharing in learning networks: a cognitive load perspective', Int. J. Continuing Engineering Education and Life-Long Learning, Vol. X, No. Y, pp.xxx-xxx.

Biographical notes: Ya Ping (Amy) Hsiao is a PhD candidate at the Research Centre for Learning, Teaching and Technology (Welten Institute) of the Open University of the Netherlands. She researches how to support learners in knowledge sharing on complex tasks. In particular, she focuses on how to facilitate peer tutoring process to achieve more efficient learning performance.

Francis Brouns is an Assistant Professor at the Research Centre for Learning, Teaching and Technology (Welten Institute) of the Open University of the Netherlands. Her current research focuses on new and innovative learning environments to support lifelong learners, in particular the learner support, social aspects of learning networks, and MOOCs. 
Peter B. Sloep is a Full Professor of Technology Enhanced Learning at the Research Centre for Learning, Teaching and Technology (Welten Institute) of the Open University of the Netherlands. His research focuses on (lifelong) learning, knowledge sharing and innovation in online environments for professionals.

This paper is a revised and expanded version of a paper entitled 'Designing optimal peer support to alleviate learner cognitive load in learning networks' presented at IADIS International Conference Web-based Communities and Social Media, Lisbon, Portugal, 19-21 July 2012.

\section{Introduction}

Learning networks (LNs) are a particular kind of online social network designed to support self-directed lifelong learners in a particular domain. They comprise groups of people who use learning resources to learn at the place, time and pace that suits them best (Koper et al., 2005; Sloep, 2009). Within our notion of an LN, learners have to take responsibility to organise their own learning activities, to acquire knowledge from others, and to achieve their personalised learning goals (Kester et al., 2007). During the learning process, it is likely that learners in LNs have the same needs as formal learners: they need to share and construct knowledge through interaction with others. In formal learning settings, this is usually done by either consulting the teacher or sharing knowledge with other students within the social structure of a class. In LNs, learners have to organise knowledge sharing themselves, and according to cognitive load theory (CLT) (Sweller, 1988), this might affect learning performance and efficiency.

CLT aims to build up pedagogical guidelines for designing learning tasks or arranging learning environments; it is based on the premise that the human cognitive architecture consists of a limited working memory and unlimited long-term memory capacities. Cognitive load refers to the learner's limited working memory capacity actually allocated to performing a particular task. It has been recognised as an important limiting factor for learner performance (Paas and Van Merriënboer, 1994; Sweller et al., 1998). CLT distinguishes three types of cognitive load: intrinsic, extraneous, and germane load (Paas et al., 2003; Van Gog and Paas, 2008). Intrinsic load is determined by the extent of element interactivity intrinsic to the learning task: a task is complex when learners have to simultaneously process multiple information elements (Sweller, 2006). Extraneous and germane load result from the design of learning tasks or environments: inefficient or bad designs impose extraneous load because learners have to allocate working memory capacity to activities that are irrelevant to learning; in contrast, efficient or good designs impose germane load because they guide learners to devote more cognitive processing to learning. For any task that learners perform, the sum of these three types of cognitive load should not overload their limited working memory capacity.

Unlike in most formal educational settings, in LNs there is no social structure of a class, nor do participants share a common learning history: they often do not even know who others are and what others know (Sloep and Berlanga, 2011). When learners have to 
self-organise knowledge sharing, they first have to find out who the potential sharers of relevant knowledge are. Then, during knowledge sharing, they have to maintain social interaction until a shared understanding is reached. These activities will increase extraneous load, because they are not directly related to learning itself but to the learning environment; that is, they detract from learning rather than facilitate it. According to CLT, extraneous load is not only ineffective or detrimental to learning but it in particular negatively impacts learning complex tasks that are already high in intrinsic load (Van Gog and Paas, 2008). For complex tasks, it is thus imperative to reduce extraneous load (Van Merriënboer and Sweller, 2005). This pedagogical guideline is particularly important in LNs. There, lifelong learners routinely encounter authentic, often complex, problems that require learners to simultaneously deal with multiple interactive information elements across different domains or situations. The extraneous load imposed by self-organised knowledge sharing plus the high intrinsic load of complex tasks, would easily overload learners' limited cognitive capacity (Hsiao et al., 2013).

To prevent this, we propose to use a peer support system (PSS) (Hsiao et al., 2013; Van Rosmalen et al., 2008a, 2008b). Such a system aims to replace the arrangements teachers in formal educational settings make to structure peer learning. In LNs, involving peers to help each other is closely akin to a commonly applied pedagogical method: peer tutoring. It is defined as "people from similar social groupings who are not professional teachers, helping each other to learn, and learning themselves by teaching" [Topping, (1996), p.322]. Conventionally, when applying peer tutoring the teacher usually relies on instructional considerations to assign peer tutors and to provide students with interaction structures that not only guide communication processes but also stimulate students to discuss their learning and negotiate meaning with each other (Dillenbourg, 1999; Topping, 1996). In our previous studies, when knowledge sharing was initiated by a tutee (i.e., a learner who asks for help) the PSS automatically assigned suitable peer tutors (i.e., learners who provide help). The PSS based itself on a set of selection criteria such as learner availability, content competency, tutor competency and eligibility (Van Rosmalen et al., 2008a, 2008b). Moreover, the PSS was equipped with instructions akin to interaction structures, which consisted of role specifications that specified what tutors and tutees should do, as well as a wiki that allowed learners to work collaboratively. This PSS, we surmise, has the potential to decrease extraneous load in two stages of self-organised knowledge sharing: finding relevant tutors and maintaining social interaction.

Therefore, the main purpose of this study is to investigate whether such a PSS can reduce extraneous load, especially when working on complex tasks. We focus on three forms of knowledge sharing support: no support (the control groups), a forum that only supports social interaction and our PSS that also supports the stage of locating relevant peers (Van Rosmalen et al., 2008a, 2008b). Since the PSS is supposed to be particularly needed for complex problems, we look at both simple and complex tasks. Thus, we aim to answer the following research question:

What are the effects of using different forms of support on learners' cognitive load and learning efficiency when working on simple and complex tasks? 
Based on the design guideline of CLT, we formulate the hypothesis as:

There is a significant interaction effect on learners' cognitive load and learning efficiency between using different forms of support and task complexity. For simple tasks, no differences of these two measures will be found between different forms of support; for complex tasks, learners supported by PSS have lower cognitive load and higher learning efficiency than forum and control groups.

\section{Method}

\subsection{Online course internet basics}

To implement this study, we set up an online course, internet basics, in a Moodle learning environment. This course was not part of any formal program of the university, so participants did not receive any European credits after they finished the course. In addition, the course was designed fully for self-study and there was no staff teacher available to steer the learning process. The estimated number of study hours was 20 . The course was available online for eight weeks. Participants who met most requirements of this course received a participation certificate.

The course consisted of general information and ten modules dealing with different subjects related to internet. The general information contains welcome words, four questions about the participant's personal situation, a 'how to complete each module', user manuals, links to internet vocabulary dictionaries, frequently asked questions (FAQ), as well as general conditions for receiving a participation certificate. The user manual included instructions on how to log onto the course site, a course overview, navigation to the course or module page, how to start with a new module and a FAQ. The FAQ consisted of three types of questions: general questions, questions about the course and questions related to computers.

Each module started with a brief introduction and consisted of several sub-topics. The ten modules were: getting more out of your internet browsers, paying safely on the internet, making use of useful websites, Web 2.0 a new internet, using the internet as a recreation place, getting access to the internet, searching the web, virus and spyware, spam and inappropriate content, and how to build a personal webpage.

\subsection{Design and participants}

Our study followed a factorial design with two between-subjects independent variables: task complexity (simple vs. complex) and types of support (control vs. forum vs. PSS). There were thus six cells; a different group of participants was allocated to each cell (see Table 1). So, although all participants had access to the same module content, each group had either simple or complex tasks or experienced a different kind of support when working on the tasks.

Through an online announcement on various websites, we recruited 534 participants and randomly assigned them to each group. However, only 329 participants actually started with the course, out of which only 167 worked on one or more tasks. The number of participants used for the analyses is therefore 167, Table 1 shows the number of participants per group. 
More than half of the 167 participants were female (61\%) and around two-thirds of the participants were older than 45 years old (68\%). $68 \%$ of the participants had a degree in higher education. More than half (57\%) of the participants indicated that they had sufficient computer skills.

Table 1 Factorial design and number of participants per cell

\begin{tabular}{lcccc}
\hline \multirow{2}{*}{ Task complexity } & \multicolumn{3}{c}{ Types of support } & \multirow{2}{*}{ Total } \\
\cline { 2 - 4 } & Control & Forum & PSS & \\
\hline Simple & 29 & 25 & 26 & 80 \\
Complex & 31 & 27 & 29 & 87 \\
Total & 60 & 52 & 55 & 167 \\
\hline
\end{tabular}

\subsection{Interventions}

\subsubsection{Task complexity}

We designed 20 tasks based on Sweller and Chandler's (1994) definition of task complexity. These tasks aimed to review the sub-topics within each module. A simple task required content knowledge of non-interacting topics (e.g., What is browser history? What is the purpose of cookies?), whereas a complex task required synthesised/integrated knowledge of multiple interacting topics (e.g., What are the differences between browser history and cookies?). Two experts in the field of CLT judged the relative complexity of these 20 tasks to ensure that task complexity was as intended.

\subsubsection{Types of support}

When working on the tasks, participants received one of the three types of support when sharing knowledge with others.

Participants in the two control groups received no support at all (nor where provided with communication means). For the forum and PSS groups, participants could submit their inquiries for knowledge sharing to a dialog box called pose your question, which for them was added to the right-hand side of each module's homepage. To submit an inquiry, participants simply clicked on Pose your question and the system navigated them to either the forum or the PSS pages. Participants in the forum groups then had to wait until others responded to their posts (as in regular forums). For PSS groups, when tutees submitted inquiries to the system, it selected available peer tutors for them. Availability was based on participants' past workload, i.e., the number of inquiries responded to previously (Van Rosmalen et al., 2008a, 2008b). To select potential peer tutors, the PSS sent out invitations that included the question posed. When someone accepted the invitation to act as peer tutor, the system would set up a wiki page private to the tutee and tutor, which contained the question and role specifications as guidelines. Peer tutor and tutee discussed and formulated answers in the wiki. When the tutee was satisfied with the answer, he or she could close the discussion and rate the answer quality. For forum and PSS groups, there was an extra manual which explained how to use the forum or PSS, respectively. 


\subsection{Measures}

\subsubsection{Cognitive load measures}

To measure cognitive load, we asked participants to report how much mental effort they invested when working on the tasks by rating on a nine-point unidimensional cognitive load rating scale (Paas, 1992; Paas et al., 2003). This rating scale ranged from a very very low effort (1) to a very very high effort (9).

\subsubsection{Pre- and post-tests}

Every module started with a pre-test and ended with a post-test. Both tests were identical and they consisted of a few content-related multiple choice questions or matching questions. The total score of each pre- and post-test was 10 . We used pre-test scores as co-variate to establish the level of similarity between the six groups of participants and post-test scores to represent learning performance.

\subsubsection{Learning efficiency}

The above-mentioned mental effort measure does not distinguish between the three types of cognitive load discussed (Beckmann, 2010; De Jong, 2010). To measure the effects of an instructional design on cognitive load, the conventional approach of most CLT studies is to combine cognitive load measures with performance measures to calculate learning efficiency (Paas and Van Merriënboer, 1993; Van Gog and Paas, 2008). Thus, a high learning efficiency can be inferred from a high performance score with a low mental effort investment, whereas a low learning efficiency is a low performance score with a high mental effort investment. Depending on the aim of an instructional design, mental effort can be measured at either the learning or testing phase for calculating two types of efficiency scores. If an instructional design aims to reduce extraneous load, the perceived mental effort at the learning phase should be combined with the performance score at the testing phase (i.e., the adapted formula); if the aim is to optimise cognitive load (to both reduce extraneous load and induce germane load), then both the mental effort and performance score at the testing phases are combined to calculate the efficiency (i.e., the original formula) (Van Gog and Paas, 2008). Since our aim is to reduce extraneous load, we combined mental effort invested in carrying out the tasks during the learning phase $(M E)$ with learning performance $(P)$ (i.e., post-test scores) to calculate adapted efficiencies $(E)$ (Van Gog and Paas, 2008). To calculate $E$, all scores of performance and mental effort were converted into standardised z-scores by first subtracting the grand mean from each score and dividing the result by the overall standard deviation, and then applying the following formula (Paas et al., 2003):

$$
E=\left(z_{P}-z_{M E}\right) / \sqrt{2}
$$

\subsubsection{Course evaluation questionnaire}

To investigate how participants appreciated using forums or PSS for sharing knowledge, we used a modified version of the course evaluation questionnaire developed by Van Rosmalen et al. (2008a). The questionnaire included the following sections: general questions (these were the same for control, forum and PSS groups), use of forums or PSS 
as being tutees and tutors, overall evaluation (only for forum and PSS groups), and closure (i.e., whether the participants wanted to apply for the certificate and research results).

\subsection{Procedure}

When the course started, participants were shown the general information when they first logged into the course site. To start with each module, participants first had to take the pre-test of that module and get an enrolment key. There were separate enrolment keys for each of the modules. Participants could only start with the module by entering the enrolment key. To complete each module, participants worked on the tasks, shared knowledge with others indicated mental effort they invested on the task as well as took the post-test. When the course was finished, we asked participants to fill in the course evaluation questionnaire by e-mail.

\subsection{Data collection during the knowledge sharing process}

To study how the PSS or the forum supports the process of knowledge sharing, we collected the following data: number of inquiries submitted for each of the modules, names of persons who submitted inquiries, names of persons who responded to the inquiries as well as the text of the inquiries and responses. In addition, for the PSS groups we traced for which topic the PSS support was activated, who was invited, how many people were invited, whether the invitation was accepted or not, and finally the outcome, as rated by the learner submitting the inquiry.

\section{Data processing and analysis}

The results show that participants did not complete all requirements that we asked them to do in general information, in particular taking the pre-tests, working on the tasks, and taking the post-tests. Only 58\% of the 167 participants took all ten pre-tests, $14 \%$ completed all ten tasks, and $53 \%$ took all ten post-tests. On average, participants took 7.46 pre-tests, completed 4.87 tasks, and took 6.79 post-tests. This shows that there were many missing values in our dataset, making statistical analysis based on the raw scores infeasible. Therefore, instead of using raw scores, we computed the means of each dependent variable (pre-tests, mental effort, and post-tests) on the modules each participant completed (see Appendix).

A two-way ANCOVA was undertaken in SPSS using the generalised linear model (GLM). We examined group differences of two independent variables (task complexity and types of support) on the means of mental effort and efficiency scores while controlling for a covariate of the means of the pre-test scores. Two assumptions underlying ANCOVA were examined: independence of the independent variables and covariate, and homogeneity of regression slopes. The test results show that these assumptions were met. A significance level of 0.05 was used for all analyses. We used partial eta-squared $\left(\eta_{p}^{2}\right)$ as an estimate of effect size, with 0.01 corresponding to a small effect, 0.06 to a medium effect, and 0.14 to a large effect (Tabachnick and Fidell, 2007). 


\section{Results}

\subsection{Mental effort}

Table 2 shows the means and standard deviations of the means of mental effort per experimental group, per independent variable and for all participants.

The test results showed that the covariate, pre-test scores, was not significantly related to mental effort scores, $F(1,160)=0.95, p=0.330$. After controlling for the effect of pre-test scores, the main effect of using different levels of support on post-test scores was non-significant, $F(2,160)=0.62, p=0.540$. However, there was a significant main effect for task complexity, $F(1,160)=4.62, p=0.033, \eta_{p}^{2}=0.03$, such that the average mental effort was higher for complex tasks than for simple tasks. There was a significant interaction effect between levels of support and task complexity on mental effort invested on the tasks, $F(2,160)=6.93, p=0.001, \eta_{p}^{2}=0.08$. This indicates that task complexity had a medium effect on mental effort depending on levels of support.

To further investigate the interaction effect we performed a simple effects analysis. The results showed that there was no significant difference in mental effort on simple tasks between the three types of support, $F(2,160)=2.71, p=0.070$. However, for complex tasks, we did find a significant difference, $F(2,160)=4.93, p=0.008$. Specifically, the results of pairwise comparison showed that on complex tasks mental effort reported by the control group was significantly lower than that reported by PSS $(p=0.005)$ and forum group $(p=0.014)$.

Table 2 Means (M) and standard deviations (SD) of means of mental effort (range: 1-9) on the tasks

\begin{tabular}{|c|c|c|c|c|c|c|c|c|}
\hline \multirow{3}{*}{$\begin{array}{l}\text { Task } \\
\text { complexity }\end{array}$} & \multicolumn{6}{|c|}{ Types of support } & \multirow{2}{*}{\multicolumn{2}{|c|}{ Total }} \\
\hline & \multicolumn{2}{|c|}{ Control } & \multicolumn{2}{|c|}{ Forum } & \multicolumn{2}{|c|}{$P S S$} & & \\
\hline & $M$ & $S D$ & $M$ & $S D$ & $M$ & $S D$ & $M$ & $S D$ \\
\hline Simple & 4.60 & 0.95 & 3.85 & 1.49 & 4.14 & 1.04 & 2.22 & 1.20 \\
\hline Complex & 4.06 & 1.48 & 4.81 & 0.98 & 4.94 & 1.10 & 4.58 & 1.27 \\
\hline Total & 4.32 & 1.27 & 4.35 & 1.33 & 4.56 & 1.13 & 4.41 & 1.24 \\
\hline
\end{tabular}

\subsection{Efficiency}

Table 3 shows the means and standard deviations of efficiency scores per experimental group, per independent variable and for all participants. According to Sweller et al. (2011), a positive efficiency score $(E>0)$ represents efficient learning (i.e., a relatively high post-test score in combination with a relatively low mental effort) whereas a negative efficiency score $(E<0)$ represents inefficient learning (i.e., a relatively low post-test score in combination with a relatively high mental effort). Table 3 shows:

1 participants in groups of complex tasks worked less efficiently than those in groups of simple tasks

2 participants in the control and PSS groups worked less efficiently than those in the forum group. 
The test results showed that the covariate, pre-test scores, was significantly related to efficiency scores, $F(1,160)=22.02, \mathrm{p}<0.001, \eta_{p}^{2}=0.12$. After controlling for the effects of pre-test scores, the main effect of using different support on efficiency scores was non-significant, $F(2,160)=1.15, p=0.320$. However, there was a significant main effect for task complexity, $F(1,160)=4.80, p=0.030, \eta_{p}^{2}=0.03$, such that the average efficiency score was higher for simple tasks than for complex tasks. The interaction effect was significant, $F(2,160)=5.29, p=0.006, \eta_{p}^{2}=0.06$, indicating task complexity had a medium effect on efficiency scores depending on levels of support.

As with learning efficiency, the results of simple effects analysis showed no significant difference in efficiency scores on simple tasks between the three types of support, $F(2,160)=2.38, p=0.096$. However, for complex tasks the difference was significant, $F(2,160)=4.03, p=0.020$. Specifically, the results of pairwise comparison showed that efficiency scores of the control group were significantly higher than those of the PSS group on complex tasks $(p=0.031)$.

Table 3 Means (M) and standard deviations (SD) of efficiency scores

\begin{tabular}{|c|c|c|c|c|c|c|c|c|}
\hline \multirow{3}{*}{$\begin{array}{l}\text { Task } \\
\text { complexity }\end{array}$} & \multicolumn{6}{|c|}{ Types of support } & \multirow{2}{*}{\multicolumn{2}{|c|}{ Total }} \\
\hline & \multicolumn{2}{|c|}{ Control } & \multicolumn{2}{|c|}{ Forum } & \multicolumn{2}{|c|}{ PSS } & & \\
\hline & $M$ & $S D$ & $M$ & $S D$ & $M$ & $S D$ & $M$ & $S D$ \\
\hline Simple & -0.23 & 1.15 & 0.46 & 1.02 & 0.33 & 0.58 & 0.17 & 0.99 \\
\hline Complex & 0.04 & 1.22 & -0.07 & 0.89 & -0.45 & 0.99 & -0.16 & 1.06 \\
\hline Total & -0.09 & 1.19 & 0.19 & 0.98 & -0.08 & 0.90 & -0.001 & 1.04 \\
\hline
\end{tabular}

\subsection{Knowledge sharing (forum and PSS groups)}

Table 4 shows that there were only a limited number of inquiries submitted by both forum and PSS groups. Therefore, we cannot fully test the hypothesis since the support of forum and PSS was not used sufficiently to have effects on knowledge sharing. In addition, Table 4 shows that only half of the inquiries received responses in the forum groups, and less than half of the inquiries were taken up in the PSS groups. Among these responses, only a small proportion of responses provided valid answers to the inquiries. Table 4 displays that groups with simple tasks submitted more inquiries than complex ones.

Table 4 Absolute frequencies and percentages of using the 'Pose your question' dialog box

\begin{tabular}{lcccc}
\hline & $\begin{array}{c}\text { Forum } \\
\text { simple }\end{array}$ & $\begin{array}{c}\text { Forum } \\
\text { complex }\end{array}$ & $\begin{array}{c}\text { PSS } \\
\text { simple }\end{array}$ & $\begin{array}{c}\text { PSS } \\
\text { complex }\end{array}$ \\
\hline Total inquiries submitted & 16 & 13 & 16 & 9 \\
Invitations sent & - & - & 58 & 34 \\
Responses provided/invitations accepted & 9 & 9 & 7 & 3 \\
Percentage responses/inquiries & $56 \%$ & $69 \%$ & $44 \%$ & $33 \%$ \\
Valid answers & 5 & 6 & 3 & 1 \\
Percentage valid answers/inquiries & $31 \%$ & $46 \%$ & $19 \%$ & $11 \%$ \\
\hline
\end{tabular}

Note: Simple refers to participants working on a simple task, complex to participants working on a complex task. 


\section{Discussion}

To summarise, though the findings showed no main effects on levels of support, they confirmed our assumption that task complexity has effects on cognitive load that learners experience. Even when working on complex tasks, mental effort was low and efficiency scores were high. This indicates that mental effort did not reach the full limit and any extraneous load there was, did not result in cognitively overloading working memory. Results also show that levels of support become important for complex tasks, as we observed an interaction effect. However, contrary to our hypothesis, the interaction effect was reversed: the control group experienced less mental effort and higher efficiency than did the forum and PSS. We will try to explain why this might have happened.

The study was designed to investigate whether extraneous cognitive load can be reduced by applying our PSS as a support structure. Unfortunately, the participants did not use the supports of forum and PSS as intended. Therefore we cannot draw any conclusions with regard to the use of the tools on cognitive load, learning performance and efficiency. One may argue that the treatments actually involved more than the mere presence of the communication tools. The treatments involved many steps the learners had to take: to sign up to Moodle, read the general information and FAQ, take as many pre-tests as they wanted to start with modules, read the instructions, read the learning materials, do assignments in the material, perform the assigned tasks, complete the mental effort instruments and take a post-test to complete the module. In addition, the forum and PSS groups received extra instructions about how to use the tools to share knowledge with others. It is possible that merely being instructed to use the tools might already have imposed too much cognitive load on the participants in addition to the fixed deadline to actually use the tools. So our argument then would be that the treatments themselves involved a significant amount of cognitive load, drowning out any effect of the cognitive load reduction we anticipated to measure.

There are other factors that might have affected the results. The evaluation questionnaire provides some insights into these. Half (53\%) of the respondents thought that the course was very easy or easy for self-study. More than three-quarter (77\%) said they were not in need of finding someone to share knowledge. These results might explain why the supports of forum and PSS were not used much, as participants did not need to share knowledge for learning in this course. This contrasts with earlier studies, in which we used the same course and a sufficient number of questions were asked (De Bakker, 2010; Van Rosmalen et al., 2008a). It might be that our participants were of a different background, or that the general knowledge about internet had increased over the years. Oddly, the level of the course was not too basic and met the learning objectives of the participants, as $70 \%$ of the respondents indicated to be satisfied with the course. Apparently they could meet their learning needs, though knowledge sharing did not occur very often. Furthermore, and importantly for our understanding of a reversed interaction effect, respondents of the control groups (85\%) were more satisfied with the course than those of the forum and PSS groups (63\%). If there is no need for knowledge sharing, those who are instructed yet to do so (forum and PSS groups), are likely to experience this as superfluous activity.

If the tasks were not complex enough to trigger needs of knowledge sharing, the setting of the control group is likely to make participants experience less workload, because they thought they just had to work alone to finish the course. This is in contrast with forum and PSS groups, whose participants were aware that they had to interact with 
others while, as argued, they actually did not need it. In particular, learners of the PSS groups might have constantly received invitations to help others' questions and this might result in higher extraneous load for two reasons. First, they might be not clear about why they received such invitations. From e-mail communications with the helpdesk we set up, we noted that many participants did not read the general information and thus did not understand that helping others' questions was part of the course requirements. Second, the PSS support in this study randomly assigned other available participants as peer tutors to those who submitted inquiries. According to e-mails we received, some participants were irritated when they received invitations because they had not yet studied the modules related to the inquiries submitted. Thus, random matching might impose extraneous load instead of reducing it.

It is interesting to note that complex tasks did not induce more knowledge sharing inquiries than the simple ones, though they did impose more cognitive load than the simple ones. One possible explanation may be that learners did not really work on these complex tasks since they did not have an obligation to do so. With estimation of mental effort in mind, they are likely to avoid being overloaded by further using the supports for knowledge sharing: to formulate their inquiries and to interact with others. To solve their problems, participants might simply have used search engines or other resources when working on their accord. We did not enforce the use of the support tools we devised, because we tried to emulate a networked learning situation as closely as possible. In such situations it is the learner who has control and decides what learning activities to perform. We hoped that by designing the course modules and the tasks carefully, the need for knowledge sharing would become apparent. Our aim was to know whether the PSS support would reduce extraneous load imposed by knowledge sharing on complex tasks: if the extraneous load is reduced, then there should be a higher efficiency score that combines low mental effort on the tasks and high learning performance scores. However, the non-formal learning environments of LNs prevented us from measuring cognitive load accurately and timely, and we could not interpret the results in the conventional way that is used in the most CLT studies. For most CLT studies, cognitive load is measured in strictly-controlled settings: participants have to complete tasks in the learning phase and then take performance tests within the limited time for every participant. In LNs, we could not force participants to fulfil all course requirements, because this would go against their self-directness, personalised learning goals and defining features of LNs. It was likely that participants chose the topics that interested them or skipped the topics they already knew.

Moreover, learners who were first-time participants in an LN are likely more interested in finding out the information or issues related to the topic and they might not be ready to ask questions (Ferguson, 2011). In addition, as indicated by help-seeking studies in online learning environments asking questions is a complex process and it can be seen as task of its own for learners (Stahl and Bromme, 2009). They need to be aware of their need for asking questions, decide to ask questions, find potential answer-givers, use strategies to ask questions and evaluate whether the answers are useful for their questions (Nelson-Le Gall, 1981; Newman, 1994). The experimental design used in this study, in which we aimed at a high ecological validity, forced us to only focus on how to support finding potential answer-givers of the entire complex process.

We conclude that there is a need to reconsider the use of conventional instruments of measuring cognitive load in open settings such as an LN environment. A corollary to this 
is that, although CLT should be applicable to less controlled situations than those investigated by cognitive load theorists to date, CLT's measurement tools are unfit for situations in which students have a high degree of self-directedness. Any future studies in ecologically valid settings, therefore, should pay much attention to developing instruments to measure cognitive load that are appropriate to the situation at hand. In addition, it turned out to be very difficult to study the various factors we believe are relevant for a support structure that has to stimulate and promote knowledge sharing in an LN context. Future studies on LNs, therefore may consider applying design-based research methods as they suit the real-world context of LNs better.

\section{References}

Beckmann, J.F. (2010) 'Taming a beast of burden - on some issues with the conceptualisation and operationalisation of cognitive load', Learning and Instruction, Vol. 20, No. 3, pp.250-264.

De Bakker, G. (2010) Allocated Online Reciprocal Peer Support via Instant Messaging as a Candidate for Decreasing the Tutoring Load of Teachers, Doctoral dissertation, Eindhoven University of Technology, Eindhoven, The Netherlands [online] https://pure.tue.nl/ws/files/3307494/685255.pdf (accessed 19 August 2016).

De Jong, T. (2010) 'Cognitive load theory, educational research, and instructional design: Some food for thought', Instructional Science, Vol. 38, No. 2, pp.105-134.

Dillenbourg, P. (1999) 'What do you mean by collaborative learning?', in Dillenbourg, P. (Ed.): Collaborative Learning: Cognitive and Computational Approaches, pp.1-19, Elsevier, Oxford, England.

Ferguson, R. (2011) 'Use of questions to facilitate social learning in a Web 2.0 environment', Globalisation and Internationalisation of Higher Education, Vol. 8, No. 1, pp.316-327.

Hsiao, Y.P., Brouns, F., Kester, L. and Sloep, P.B. (2013) 'Cognitive load and knowledge sharing in learning networks', Interactive Learning Environments, Vol. 21, No. 1, pp.89-100, DOI: $10.1080 / 10494820.2010 .548068$.

Kester, L., Van Rosmalen, P., Sloep, P.B., Brouns, F., Koné, M. and Koper, R. (2007) 'Matchmaking in learning networks: bringing learners together for knowledge sharing', Interactive Learning Environments, Vol. 15, No. 2, pp.117-126.

Koper, R., Giesbers, B., van Rosmalen, P., Sloep, P.B., van Bruggen, J., Tattersall, C. and Brouns, F. (2005) 'A design model for lifelong learning networks', Interactive Learning Environments, Vol. 13, No. 1, pp.71-92.

Nelson-Le Gall, S. (1981) 'Help-seeking: an understudied problem-solving skill in children', Developmental Review, Vol. 1, No. 3, pp.224-246.

Newman, R.S. (1994) 'Adaptive help seeking: a strategy of self-regulated learning', in Schunk, D.H. and Zimmerman, B.J. (Eds.): Self-regulation of Learning and Performance: Issues and Educational Applications, pp.283-301, Erlbaum, Hillsdale, NJ.

Paas, F. (1992) 'Training strategies for attaining transfer of problem-solving skill in statistics: a cognitive-load approach', Journal of Educational Psychology, Vol. 84, No. 4, pp.429-434.

Paas, F. and Van Merriënboer, J. (1993) 'The efficiency of instructional conditions: an approach to combine mental effort and performance measures', Human Factors, Vol. 35, No. 4, pp.737-743.

Paas, F. and Van Merriënboer, J. (1994) 'Instructional control of cognitive load in the training of complex cognitive tasks', Educational Psychology Review, Vol. 6, No. 4, pp.351-371.

Paas, F., Renkl, A. and Sweller, J. (2003) 'Cognitive load theory and instructional design: recent developments', Educational Psychologist, Vol. 38, No. 1, pp.1-4.

Paas, F., Tuovinen, J., Tabbers, H. and Van Gerven, P. (2003) 'Cognitive load measurement as a means to advance cognitive load theory’, Educational Psychologist, Vol. 38, No. 1, pp.63-71. 
Sloep, P.B. (2009) 'Fostering sociability in learning networks through ad-hoc transient communities', in Purvis, M. and Savarimuthu, B.T.R. (Eds.): Computer-Mediated Social Networking, pp.62-75, Springer, Heidelberg, Germany.

Sloep, P.B. and Berlanga, A.J. (2011) 'Learning networks, networked learning [Redes de Aprendizaje, Aprendizaje en Red]', Comunicar., Vol. 19, No. 37, pp.55-63.

Stahl, E. and Bromme, R. (2009) 'Not everybody needs help to seek help: surprising effects of metacognitive instructions to foster help-seeking in an online-learning environment', Computers \& Education, Vol. 53, No. 4, pp.1020-1028.

Sweller, J. (1988) 'Cognitive load during problem solving: effects on learning', Cognitive Science, Vol. 12, No. 2, pp.257-285.

Sweller, J. (2006) 'How the human cognitive system deals with complexity', in Elen, J. and Clark, R.E. (Eds.): Handling Complexity in Learning Environments: Theory and Research, pp.13-25, Elsevier, Oxford, England.

Sweller, J. and Chandler, P. (1994) 'Why some material is difficult to learn', Cognition and Instruction, Vol. 12, No. 3, pp.185-233.

Sweller, J., Ayres, P. and Kalyuga, S. (2011) 'Measuring cognitive load', in Sweller, J., Ayres, P. and Kalyuga, S. (Eds.): Cognitive Load Theory, pp.71-85, Springer, New York, NY.

Sweller, J., Van Merriënboer, J. and Paas, F. (1998) 'Cognitive architecture and instructional design’, Educational Psychology Review, Vol. 10, No. 3, pp.251-296.

Tabachnick, B.G. and Fidell, L.S. (2007) Using Multivariate Statistics, 5th ed., Pearson Education, Boston, MA.

Topping, K.J. (1996) 'The effectiveness of peer tutoring in further and higher education: a typology and review of the literature', Higher Education, Vol. 32, No. 3, pp.321-345.

Van Gog, T. and Paas, F. (2008) 'Instructional efficiency: revisiting the original construct in educational research', Educational Psychologist, Vol. 43, No. 1, pp.16-26.

Van Merriënboer, J. and Sweller, J. (2005) 'Cognitive load theory and complex learning: recent developments and future directions', Educational Psychology Review, Vol. 17, No. 2, pp.147-199.

Van Rosmalen, P., Sloep, P.B., Brouns, F., Kester, L., Berlanga, A., Bitter, M. and Koper, R. (2008a) 'A model for online learner support based on selecting appropriate peer tutors', Journal of Computer Assisted Learning, Vol. 24, No. 6, pp.483-493.

Van Rosmalen, P., Sloep, P.B., Kester, L., Brouns, F., De Croock, M., Pannekeet, K. and Koper, R. (2008b) 'A learner support model based on peer tutor selection', Journal of Computer Assisted Learning, Vol. 24, No. 1, pp.74-86.

\section{Appendix}

\section{Distribution of raw scores used for calculating means}

This table summarises the data on which the means of each dependent variable was based. For example, the topmost value in the pre-tests column indicates that 12 out of 167 participants (7.2\%) took the pre-test of one module. Similarly, the final value in the tasks column indicates that 23 participants (13.8\%) completed the tasks of all ten modules. 
14 Y.P. Hsiao et al.

Table A1 Frequency (percent) of the number of pre-tests taken, tasks completed and post-tests taken

\begin{tabular}{lccc}
\hline Number of modules & Pre-tests & Tasks & Post-tests \\
\hline 1 & $12(7.2 \%)$ & $47(28.1 \%)$ & $26(15.6 \%)$ \\
2 & $15(9.0 \%)$ & $21(12.6 \%)$ & $12(7.2 \%)$ \\
3 & $11(6.6 \%)$ & $8(4.8 \%)$ & $10(6.0 \%)$ \\
4 & $8(4.8 \%)$ & $11(6.6 \%)$ & $8(4.8 \%)$ \\
5 & $5(3.0 \%)$ & $5(3.0 \%)$ & $4(2.4 \%)$ \\
6 & $6(3.6 \%)$ & $10(6.0 \%)$ & 0 \\
7 & $3(1.8 \%)$ & $10(6.0 \%)$ & $5(3.0 \%)$ \\
8 & $4(2.4 \%)$ & $16(9.6 \%)$ & $3(1.8 \%)$ \\
9 & $6(3.6 \%)$ & $16(9.6 \%)$ & $7(4.2 \%)$ \\
10 & $97(58.1 \%)$ & $23(13.8 \%)$ & $88(52.7 \%)$ \\
\hline
\end{tabular}

